\title{
Correlated Nanoscopic Josephson Junctions
}

\author{
Stefano Bellucci ${ }^{1}$ Michele Cini $^{1,2}$, Pasquale Onorato ${ }^{1,3}$, and Enrico Perfetto ${ }^{1,4}$ \\ ${ }^{1}$ INFN, Laboratori Nazionali di Frascati, P.O. Box 13, 00044 Frascati, Italy. \\ 2 Dipartimento di Fisica, Università di Roma Tor Vergata, Via della Ricerca Scientifica, Roma, Italy \\ 3 Dipartimento di Scienze Fisiche, Università di Roma Tre, Via della Vasca Navale 84, 00146 Roma, Italy \\ ${ }^{4}$ Instituto de Estructura de la Materia. Consejo Superior de Investigaciones Científicas. Serrano 123, 28006 Madrid. Spain
}

(Dated: September 2, 2018)

\begin{abstract}
We discuss correlated lattice models with a time-dependent potential across a barrier and show how to implement a Josephson-junction-like behavior. The pairing occurs by a correlation effect enhanced by the symmetry of the system. In order to produce the effect we need a mild distortion which causes avoided crossings in the many-body spectrum. The Josephson-like response involves a quasi-adiabatic evolution in the time-dependent field. Besides, we observe an inverse-Josephson (Shapiro) current by applying an AC bias; a supercurrent in the absence of electromotive force can also be excited. The qualitative arguments are supported by explicit exact solutions in prototype 5 -atom clusters with on-site repulsion. These basic units are then combined in ring-shaped systems, where one of the units sits at a higher potential and works as a barrier. In this case the solution is found by mapping the low-energy Hamiltonian into an effective anisotropic Heisenberg chain. Once again, we present evidence for a superconducting flux quantization, i.e. a Josephson-junction-like behavior suggesting the build-up of an effective order parameter already in few-electron systems. Some general implications for the quantum theory of transport are also briefly discussed, stressing the nontrivial occurrence of asymptotic current oscillations for long times in the presence of bound states.
\end{abstract}

\section{INTRODUCTION}

The Josephson effect is one of the clearest fingerprints from which one can diagnose superconductivity. A DC electromotive force (e.m.f.) $V$ across a thin insulating layer in a superconductor-insulator-superconductor junction produces $\underline{\underline{1}}$ an $\mathrm{AC}$ current response. The Josephson current is given by the well known expression 2

$$
I=I_{J} \sin \left(\frac{2 e V}{\hbar} t+\gamma_{0}\right)
$$

where $I_{J}$ and $\gamma_{0}$ are constants. In textbooks this result is explained in terms of the order parameter $\psi$ at the junction; within the insulator one has

$$
\psi(z)=\psi_{\text {left }} e^{-\alpha z}+\psi_{\text {right }} e^{\alpha(z-s)},
$$

where $s$ is the barrier width, $\alpha$ is a characteristic inverse length and $z$ is the normal coordinate; $\psi_{\text {left }}$ and $\psi_{\text {right }}$ are complex constants. The quantum mechanical current $I$ is computed by the Ginzburg-Landau formula, that is, by using $\psi$ as a single-particle wave function. Thus, $I$ is proportional to $\sin \theta$, where $\theta$ is the phase difference between $\psi_{\text {left }}$ and $\psi_{\text {right }}$. Letting $\theta(t)=\frac{2 e V}{\hbar} t+\gamma_{0}$ we obtain (1).

The electrons cannot come into play in this picture, since the order parameter is a macroscopic concept. A very enlightening model argument which is microscopic in spirit is the one due to Ferrel and Prange $\stackrel{4}{\text {. Let }} H_{T}$ denote the electron tunneling Hamiltonian between the left and right superconductors. The junction with $N-\nu$ pairs on the left and $\nu$ on the right (both numbers being huge) is mapped to a tight-binding chain described by an effective Hamiltonian $H_{F}$ that describes pair hopping. For $H_{T} \rightarrow 0$ the energy $E_{0}$ of the junction does not depend on $\nu$. So, the state is defined by the amplitude $\phi_{\nu}$ obtained from

$$
H_{F} \phi_{\nu}=E_{0} \phi_{\nu}+F\left(\phi_{\nu+1}+\phi_{\nu-1}\right),
$$

where the pair hopping matrix element arising to the second-order in $H_{T}$ reads

$$
F=\sum_{i} \frac{\left\langle\nu+1\left|H_{T}\right| i\right\rangle\left\langle i\left|H_{T}\right| \nu\right\rangle}{E_{0}-E_{i}}
$$

and the sum is over the intermediate states $i$ of energy $E_{i}$ with an electron in the barrier. The eigenfunctions of $H_{F}$ are plane waves in $\nu$ space, with eigenvalues

$$
\epsilon(k)=E_{0}+2 F \cos k .
$$


A current

$$
I=\frac{4 e F}{\hbar} \sin k
$$

is associated to the group velocity $\frac{1}{\hbar} \frac{\partial E}{\partial k}$ in the fictitious chain and to the real current across the unbiased junction. A wave packet centered at $\bar{k}$ in the fictitious chain would be accelerated by

$$
\frac{d}{d t} \hbar \bar{k}=2 e V
$$

and this inserted into (6) reproduces (11) again, seen from another angle. The description by Ferrel and Prange is still aimed at a macroscopic system with huge pair numbers enabling us to use the concept of a group velocity.

The question that arises is: what happens in small systems, when the fictitious chain is very short and the group velocity has no meaning? The Ferrel - Prange description must be modified, however pairs will continue to hop as bound units, and the AC response to a DC bias is a qualitative feature which looks well suited to test any model for superconductivity. Being a qualitative feature, it is hard to see how it can depend on size. Moreover, the order parameter which is well defined only in the thermodynamic limit must be supplemented by a microscopic description valid in finite systems; there, such concepts like long-range order do not apply but clear signatures like lack of dissipation must still distinguish superconducting from normal currents. The very nature of these signatures deserves investigation.

In this paper we wish to look for precursors to the Josephson behavior far from the thermodynamic limit; small cluster studies are motivated by conceptual as well as practical reasons. Indeed, the growing technological interest in nano-systems justifies the identification and search. We focus on repulsive Hubbard-like models that we want to test for correlation-induced superconductivity; this is relevant to the search for possible non-conventional mechanisms, that have been considered by several workers e.g. in the context of high- $T_{C}$ materials. The choice of the geometry has been prompted by previous studies ${ }^{\underline{\underline{\underline{T}}}}$ in the framework of the $W=0$ theory; this develops the role of symmetry in inducing pairing from repulsive interactions. Besides, we also found the conditions leading to superconducting flux quantization in mesoscopic systems. We are therefore interested in testing this mechanism in time-dependent conditions.

Below we use a Gedanken experiment for understanding the problem. Developing this idea, one meets the difficulty that small systems always give AC response to a DC bias for quantum mechanical reasons; that is, one finds oscillating currents analogous to Rabi oscillations. However, the frequency of the oscillations is proportional to the charge, so a sharp effect of pairing remains, i.e. supercurrents are signaled by doubled frequencies. This is a conceptual problem and we shall be in position to add more to try to clarify this point in the conclusions.

There are further reasons of interest in this kind of problems. Transient current response are now actively studied, and within the TDLDA the asymptotic behavior of currents yields the static current-voltage characteristic ${ }^{7}$. Here we shall find a class of systems where, owing to correlation induced bound states, the asymptotic behavior is oscillatory. There is a clear need to develop new techniques, capable of tackling such situations.

Here we outline the plan of this paper. In Section【we summarize the theory of $W=0$ pairing in Hubbard clusters. Section IIII presents our one-unit model. Here we discuss the simplest case, namely a 5-site Hubbard cluster with square symmetry. For short, we shall refer to it as the $\mathrm{CuO}_{4}$ unit; this is known to yield pairing and flux quantization, and here we show that it is also a prototype system for dynamic phenomena. One must perturb the square symmetry, in order to mimic a barrier in such a tiny system. The current excited by a constant electro-moving force (emf) is oscillatory with a frequency which is exactly the same as the Josephson frequency in Eq. (11); moreover we shall observe Shapiro spikes (inverse Josephson effect) which represent a DC response to an AC bias at certain frequencies. A constant current (with some periodic ripple) flows in the absence of an applied potential difference, which is typical of superconducting systems. All these effects disappear when pairing fails, e.g. if the repulsion $U$ is removed. In Section IV we shall extend the investigation of Josephson-like currents to large systems that are rings composed of any number of $\mathrm{CuO}_{4}$ units, up to the thermodynamic limit in principle. For small inter-unit hopping, the many-electron system can be exactly mapped into a Heisenberg-Ising-like chain. Systems hosting many pairs are described. An effective barrier is simulated by lifting the one-body levels of one of the $\mathrm{CuO}_{4}$ clusters; the superconducting flux quantization is again obtained and time-dependent solutions with a constant emf are also presented. Finally, we present our conclusions in Section $\nabla$ 


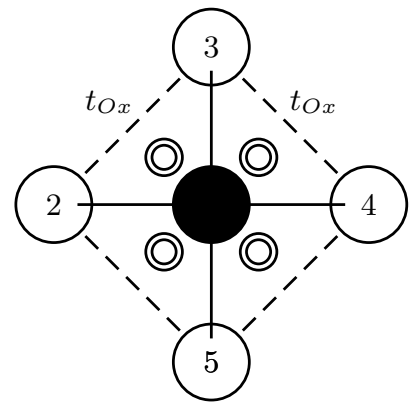

a)

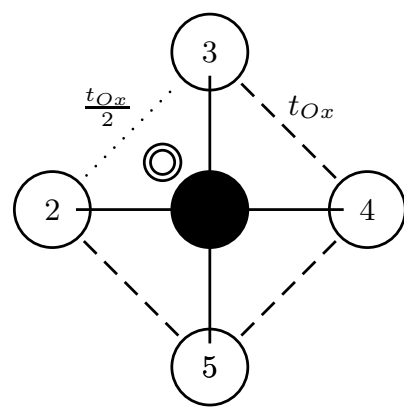

b)

FIG. 1: a) The undistorted 5-atom unit with 4 flux tubes b) The distorted 5-atom unit with 1 flux tube and reduced 2-3 hopping (the barrier).

\section{II. $\mathrm{W}=0$ PAIRING}

In the context of High- $\mathrm{T}_{C}$ superconductivity, several approaches based on the Hubbard Hamiltonian have appeared in the literature. The quantity

$$
\Delta(N)=E(N)+E(N-2)-2 E(N-1)
$$

where $E(N)$ is the ground state energy of the system with $N$ fermions is used to evaluate the effective interaction between particles in the interacting ground state. A negative $\Delta$ means that an effective attraction is developing from the repulsive interactions. We refer to Ref. 5 for the general theory based on the fact that highly-symmetric clusters possess 2-particle singlet eigenstates which do not feel the on-site repulsion $W$; these are called $W=0$ pairs. The persistence of the " $W=0$ " character in the interacting case is discussed in detail there, with the flux quantization properties and their group theory analysis. The $\mathrm{CuO}_{4}$ cluster with Hamiltonian

$$
H_{0}=\epsilon_{p} \sum_{\sigma, i=2}^{5} p_{i, \sigma}^{\dagger} p_{i, \sigma}+t_{p d} \sum_{i \sigma}\left(d_{\alpha \sigma}^{\dagger} p_{\alpha, i \sigma}+p_{\alpha, i \sigma}^{\dagger} d_{\alpha \sigma}\right)+U\left(n_{\alpha \uparrow}^{(d)} n_{\alpha \downarrow}^{(d)}+\sum_{i} n_{\alpha, i \uparrow}^{(p)} n_{\alpha, i \downarrow}^{(p)}\right),
$$

is the simplest one to give the effect; here, $p_{\sigma, i}^{\dagger}$ is the creation operator for a fermion onto the Oxygen $i=2, . ., 5, d$ destroys a fermion on the central site 1 ; besides, $\epsilon_{p}$ is the $\mathrm{O}$ energy level, $t_{p d}$ is the p-d hopping matrix element, and $U$ is the Hubbard repulsion. In a wide range of parameters, this model yields $\Delta<0$ for $N=4$.

The magnetic properties of the $\mathrm{CuO}_{4}$ cluster will be discussed below in more detail. 


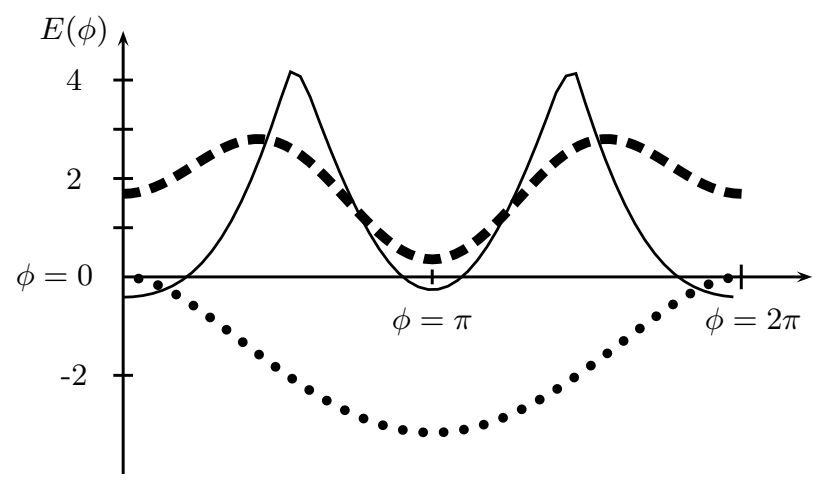

FIG. 2: The ground state energy $E(\phi)$ of the $\mathrm{CuO}_{4}$ with $N=4$ versus flux $\phi$, in $t_{p d}$ units; $t_{O x}=-0.05, \varepsilon_{p}=3.5, U=5.3$. Solid: plot of $100 *(E(\phi)-7.97)$ for the undistorted $C_{4 v}$-symmetric $\mathrm{CuO}_{4}$ cluster with equal O-O bonds and 4 equal flux tubes. Dashed: plot of $1000 *(E(\phi)-7.97)$ for the distorted cluster. Notice that we enhanced the effect 10 times in this case for easier comparison. Dotted: plot of $50 *(E(\phi)-5.126)$ for the same input data as the dashed line but $U=0$. In the absence of repulsion there is no pairing and the 4 particle system is paramagnetic.

\section{THE ONE-UNIT MODEL}

For simplicity, $\mathrm{CuO}_{4}$ clusters are natural units to build more interesting systems. We start from the simple 5-atom unit and then show how we can combine many units to build large systems in Sect. IV In order to have closed circuits where flux tubes can be inserted, we consider the time-dependent Hamiltonian (see Figure 1)

$$
H_{\text {tot }}=H_{0}+H_{\tilde{t}}(t)
$$

where

$$
H_{\tilde{t}}(t)=\sum_{i \sigma} \tilde{t}_{i, i+1} p_{i, \sigma}^{\dagger} p_{i+1, \sigma}+\text { h.c. }
$$

Henceforth $t_{p d}$ will be our energy unit; we could dispense from introducing $\epsilon_{p}$, but in this section $\epsilon_{p}=3.5$ to make the flux quantization pattern more pronounced. There are various cases to consider.

\section{A. Symmetric cluster}

Letting $\tilde{t}_{i, i+1}=t_{O x}$ we have the symmetric cluster of Figure $1 \mathrm{a}$ ). Moreover we insert flux tubes (see Figure $1 \mathrm{a}$ )) by setting $\tilde{t}_{i, i+1}=t_{O x} e^{i \gamma(t)}$ with $\gamma=\frac{2 \pi \phi}{\phi_{0}}$, where $\phi$ is the flux in each tube; the flux does not affect the $t_{p d}$ bonds by symmetry. We observe a superconducting flux quantization due to the $W=0$ pairs as shown in Figure 2, solid line. A typical double-minimum pattern is found with sharp maxima due to the level crossing of paired states with different symmetry. This patter is obtained with $t_{O x}=-0.05$; if the absolute value of the hopping $t_{O x}$ is increased too much, the coupling to the flux becomes excessive compared to the pairing energy and the system turns paramagnetic $0^{5}$. There are two minima at $\phi=\frac{\phi_{0}}{2}$ and $\phi=\phi_{0}$; in both minima, there is pairing $(\Delta<0)$. This pattern disappears together with pairing for $U=0$.

For a time-independent flux, the $\tilde{t}$ bonds carry a screening current, since $\hat{j} \propto-\frac{\partial H}{\partial \phi} 6 . \phi=\phi(t)$ is equivalent to applying an emf across each $\mathrm{O}-\mathrm{O}$ bond. In the absence of interactions, the current response to a constant emf across e.g. the 2-3 bond is periodic due to the finite system (see below). If the flux varies slowly, the system will follow the ground state curve adiabatically up to the crossing point. However since each level has conserved quantum numbers, 


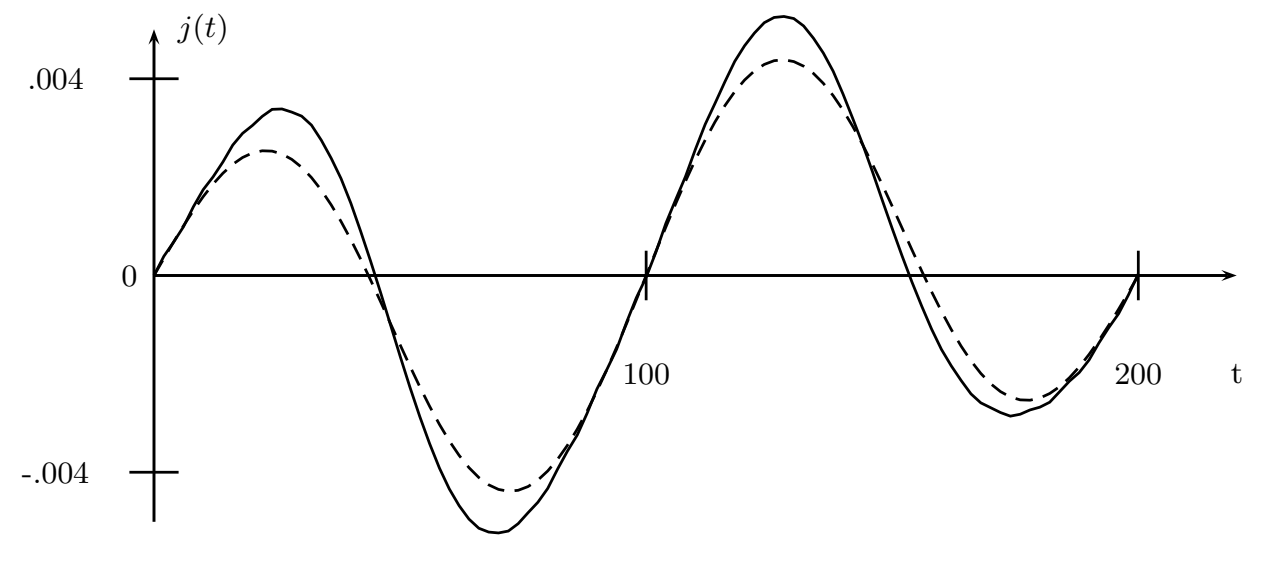

FIG. 3: The dynamical current response in units of $\frac{e t_{p d}}{\hbar}$ (solid line) versus time flowing in the $2-3$ bond with $\phi(t)=\eta t, \eta=\frac{\pi}{100}$. This value of $\eta$ is below the gap in the spectrum, which is of order $\left|t_{O x}\right|=0.05$.

the system cannot adiabatically follow the ground state with slowly increasing $\phi$. As a result, the adiabatic current is periodic with the same period as in a normal system (e.g. the cluster with $U=0$ ).

\section{B. Static properties of the mildly distorted cluster}

A more interesting behavior is obtained by breaking the square symmetry, as shown in Figure 1b). A superconducting pattern is still visible (Figure 2, dashed line). Here, $\tilde{t}_{i, i+1}=t_{o x}$ is a constant for all bonds, except for the bond $2-3$, where we take $\tilde{t}_{2,3}=\frac{t_{o x}}{2} e^{i \gamma(t)}$. No flux is present in the other O-O-Cu triangles. In Figure 3, dashed line, we see that the superconducting double minimum pattern is still there but the level crossing is avoided opening a gap in the 4-fermion spectrum. However, the repulsion is needed to produce pairing and flux quantization. The dotted line shows the drastic effect of setting $U=0$ : the system becomes paramagnetic and normal.

\section{Emf and frequency of currents}

In the paired situation of Figure 2, dashed line, we will consider the time-dependent case, $\phi / \phi_{0} \rightarrow V t$, where $V$ plays the role of an electromotive force, and $t$ in units of $\frac{\hbar}{t_{p d}}$. An adiabatic behavior is now achieved when the time dependence is slow compared to the gap. We are using the Gauss system, hence

$$
\phi_{0}=\frac{h c}{e}
$$

and the Faraday law reads

$$
V=-\frac{1}{c} \frac{\partial \phi}{\partial t} .
$$




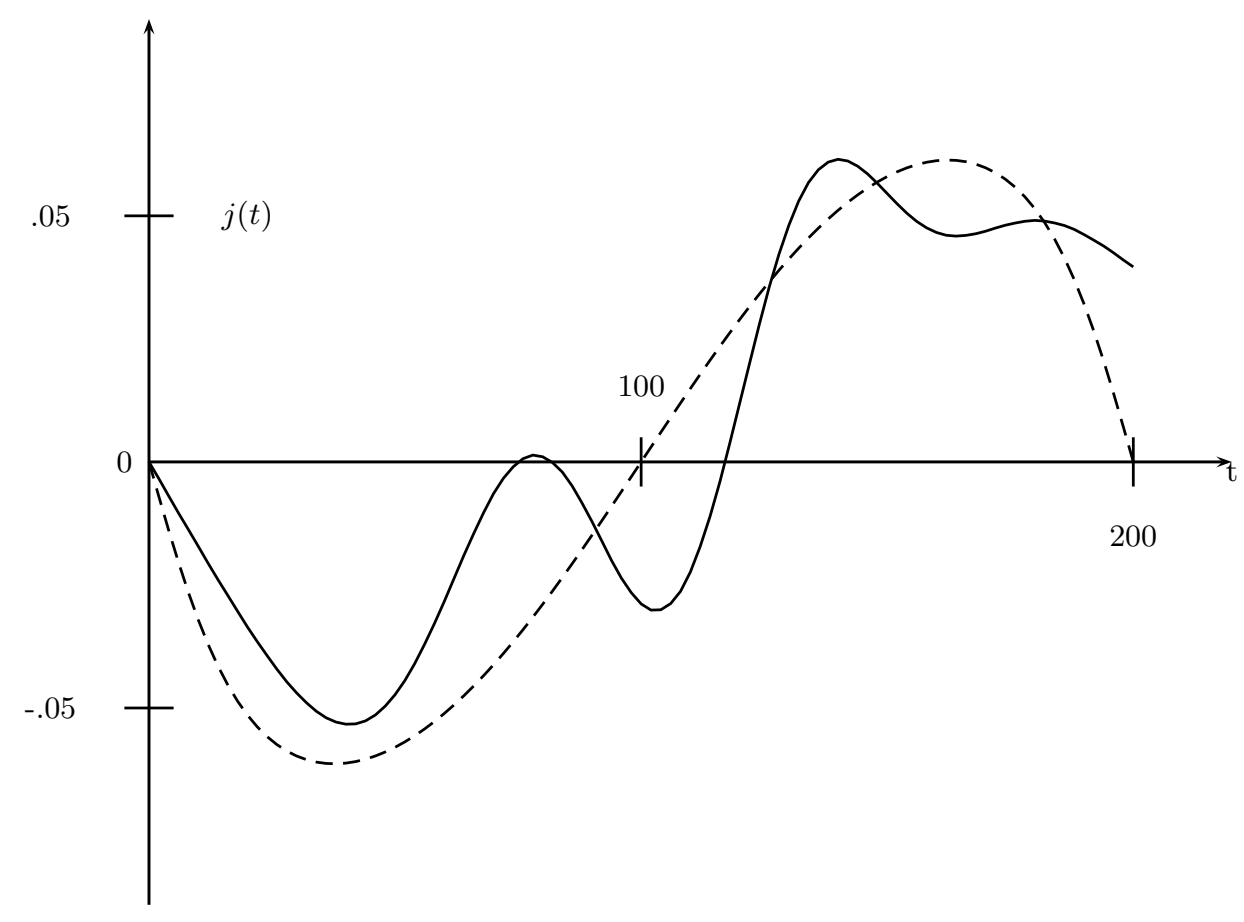

FIG. 4: The dynamical current response in units of $\frac{e t_{p d}}{\hbar}$ (solid line) versus time flowing in the $2-3$ bond with $\phi(t)=\eta t, \eta=\frac{\pi}{100}$, as above, but $U=0$.

Therefore, we introduce the frequency $\eta$ by setting

$$
\eta=-\frac{e V}{\hbar}
$$

Equation (1) becomes

$$
\frac{I}{I_{J}}=\sin (2 \eta t)
$$

for superconductor junctions, while the 2 is missing for normal systems.

This estimate agrees with the results of Figure 3. The solid line is the current through the 2-3 bond obtained by integrating the Schrödinger equation. The initial conditions are $\phi=0$ with the system in the ground state with $N=4$. The dotted line, reported for comparison, is the adiabatic response $j_{a d}(t)=c \frac{\partial E(4)}{\partial \phi(t)}$. The two curves agree fairly well. Since $t_{O x} \ll 1$, the main contribution to the current response comes from the 123 plaquette, which responds with frequency $\eta$ for normal systems and $2 \eta$ for superconducting behavior. Some current contribution involves other plaquettes as well, perhaps mixing different frequencies. The agreement with the adiabatic approximation confirms the present analysis and will be used in Sect.IV . The normal response would be periodic with half the frequency.

The system becomes normal for $U=0$, a fact which is apparent from the magnetic response (dotted line of Figure 2 ). The current response through the 2-3 bond also become normal, as we can see from the halved frequency of the current response in Figure 4. Note that the adiabatic result (dashed) does not agree very well with the response obtained by integrating the Schrödinger equation (solid). In fact, the gap $\sim t_{O x}$ is missing in the spectrum.

\section{Supercurrent flowing without bias}

In a macroscopic junction one can excite a supercurrent that will last forever if the exciting bias is removed, unless there are dissipative elements elsewhere in the circuit. This is also borne out in the paired situation of Figure 2, dashed line; we will consider the time-dependent case, $\phi / \phi_{0} \rightarrow V t$, where $V$ plays the role of an emf of finite duration. 


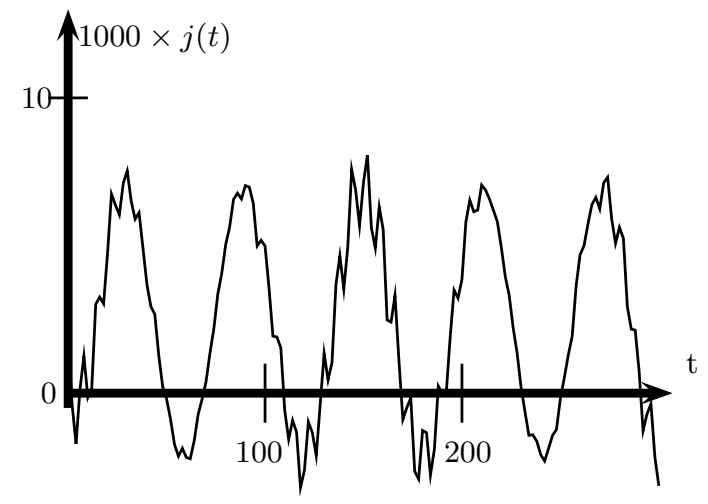

FIG. 5: Current response in units of $\frac{e t_{p d}}{\hbar}$ of the mildly distorted $\mathrm{CuO}_{4}$ unit. A constant emf across the 2-3 bond (barrier) lasts from $t=0$ to $t=20$. At $t=0$, The current response to a flux $\phi(t)=\gamma_{0}+\eta t$ where $\eta=\frac{\pi}{100}$ as above and $\gamma_{0}=\frac{\pi}{2}$. A constant supercurrent is superimposed to the oscillations.

In Figure 5 we plot the current flowing across the $2-3$ bond (barrier) when the emf lasts from $t=0$ to $t=20$. At $t=0, \phi=\frac{\pi}{2}$. A constant supercurrent is superimposed on the oscillatory response.

\section{E. Shapiro-like effect}

Still in the paired situation of Figure 2, dashed line, we also consider the time-dependent case of an oscillating field. In superconducting junctions there are spikes in the current-voltage characteristics when the voltage has a constant component and an oscillating one at radio-frequency $\omega_{r}$ of amplitude $V_{r}$ (Shapiro effect):

$$
V_{t o t}=V+V_{r} \cos \left(\omega_{r} t\right)
$$

Then Eq.(11) reads:

$$
I(t)=I_{J} \sin \left[\frac{2 e V t}{\hbar}+\gamma_{0}+\frac{2 e V_{r}}{\hbar \omega_{r}} \sin \left(\omega_{r} t\right)\right]
$$

In particular one finds a zero-voltage spike, because at $V=0$ the resulting $I(t)$ has a DC component. We can mimic such a behavior with our model. In Figure 6 we plot the current response through the 2-3 bond to a flux

$$
\phi(t)=\gamma_{0}+2 \frac{\eta_{r}}{\omega_{r}} \sin \left(\omega_{r} t\right)
$$

with $\gamma_{0}=\frac{\pi}{2}, \eta_{r}=\frac{\pi}{200}$ and $\omega_{r}=\frac{\pi}{10}$. We observe the typical Shapiro-like DC current with a ripple on it.

\section{F. Comment on the one-unit model}

It appears from the above that the 5-atom cluster with suitable parametrization can simulate all the characteristic signatures of Josephson junctions, as if it already contained a seed of the order parameter. This is indeed surprising, but we show below that combining the small units we can go smoothly to large systems still keeping the same characteristic behavior; simplicity stems from the fact that if there are many pairs they move coherently. 


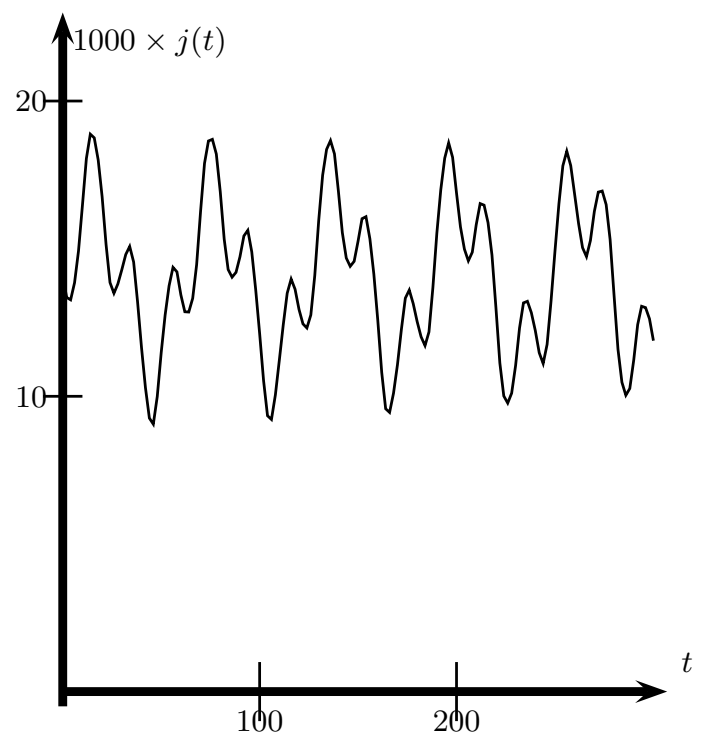

FIG. 6: The current response in units of $\frac{e t_{p d}}{\hbar}$ to a flux $\phi(t)=\gamma_{0}+\frac{2 \eta}{\omega_{r}} \sin \left(\omega_{r} t\right)$ where $\gamma_{0}=\frac{\pi}{2}$ and $\omega_{r}=\frac{\pi}{10}$. The Shapiro-like constant component is superimposed to a ripple due to the alternative paths available to the current.

\section{THE MANY-UNITS MODEL}

In This Section we study the time-dependent propagation of many $W=0$ pairs along rings of $\mathrm{CuO}_{4} \mathrm{Hubbard}$ clusters in presence of a barrier.

Our system is made of $L-1 \mathrm{CuO}_{4}$ units plus a "barrier" standing between the 1-st and the $L-1$-th units. The barrier itself is taken to be a $\mathrm{CuO}_{4}$ cluster, but with very high on-site energies, in order to make its occupancy only virtual.

The $L-1$ units are connected each other by the usual inter-cluster hopping Hamiltonian $H_{\tau}$, with real hopping integral $\tau$, while the hopping between the barrier and the 1-st and the $L-1$-th units is assumed to be complex and time-dependent: $\tau^{\prime} e^{i \gamma(t)}$, where the phase $\gamma(t)$ will be discussed later. By means of such a choice we have in mind that in transport gedankenexperiments the potential drops only through the barrier.

According to the notation of our previous work ${ }^{8}$, the model reads:

$$
H_{\text {tot }}=H_{0}+H_{\tau}+H_{\text {barrier }}+H_{\tau^{\prime}}(t)
$$

with

$$
H_{0}=\sum_{\alpha=1}^{L-1}\left[t_{p d} \sum_{i \sigma}\left(d_{\alpha \sigma}^{\dagger} p_{\alpha, i \sigma}+p_{\alpha, i \sigma}^{\dagger} d_{\alpha \sigma}\right)+U\left(n_{\alpha \uparrow}^{(d)} n_{\alpha \downarrow}^{(d)}+\sum_{i} n_{\alpha, i \uparrow}^{(p)} n_{\alpha, i \downarrow}^{(p)}\right)\right]
$$

where, $p_{\alpha, i \sigma}^{\dagger}$ is the creation operator for a hole onto the Oxygen $i=1, . ., 4$ of the $\alpha$-th cell and so on. In this section $\epsilon_{p}=0$.

$H_{\tau}$ is an inter-cell hopping Hamiltonian which allows a fermion in the $i$-th Oxygen site of the $\alpha$-th unit to move towards the $i$-th Oxygen site of the $\alpha \pm 1$-th unit (without involving the $L$-th unit, which is the barrier) with hopping 
integral $\tau$ :

$$
H_{\tau}=\sum_{\alpha=1}^{L-2} \sum_{i \sigma} \tau p_{\alpha, i \sigma}^{\dagger} p_{\alpha+1, i \sigma}+h . c . .
$$

Finally we model the barrier with the following Hamiltonian:

$$
H_{\mathrm{barrier}}=t_{p d} \sum_{i \sigma}\left(d_{L \sigma}^{\dagger} p_{L, i \sigma}+p_{L, i \sigma}^{\dagger} d_{L \sigma}\right)+U\left(n_{L \uparrow}^{(d)} n_{L \downarrow}^{(d)}+\sum_{i} n_{L, i \uparrow}^{(p)} n_{L, i \downarrow}^{(p)}\right)+W N_{t o t},
$$

where $N_{t o t}$ is the number of particles in the $L$-th cluster and $W$ is an extra (large) energy felt by each particle in the barrier, which can be provided, for instance, by a gate voltage. The barrier is linked to the rest of the ring by a complex hopping Hamiltonian:

$$
H_{\tau^{\prime}}(t)=\tau^{\prime} e^{i \gamma(t)} \sum_{i \sigma}\left(p_{L-1, i \sigma}^{\dagger} p_{L, i \sigma}+p_{L, i \sigma}^{\dagger} p_{1, i \sigma}\right)+\text { h.c. } \equiv \tau^{\prime} e^{i \gamma(t)} H_{J}+\text { h.c. }
$$

In the static case we set (with the same notation of 8 )

$$
\gamma=\frac{2 \pi \phi}{\Lambda \phi_{0}}
$$

where $\phi$ is the total flux through the ring and $\Lambda=2$ as long as the flux is associated with two bonds. In the time-dependent case, we will set $\phi / \phi_{0} \rightarrow V t$, where $V$ plays the role of an electromotive force, as in the previous Section.

\section{A. Static Case}

Let us begin with the static case. As usual we introduce $H_{\tau}+H_{\tau^{\prime}}$ perturbatively, in the same spirit of our previuos work ${ }^{8}$. When $U / t \simeq 5, \tau=\tau^{\prime}=0$ and the number of particles is appropriate and even, each $\mathrm{CuO}_{4}$ cluster is populated by 2 or 4 particles (since the occupations with 3 particles have a gap of the order of $|\Delta|$ ), while the barrier is totally empty, since the extra energy even for a single occupancy is very large. Now, if $|\tau|,\left|\tau^{\prime}\right|<<|\Delta|$, the perturbations are operative only by the second order. In particular $H_{\tau}$ provides an effective hopping $\tau_{\text {eff }} \sim \tau^{2} /|\Delta|$ for bound $W=0$ pairs, which can propagate in the $1,2, \ldots, L-1$ clusters as hard-core bosons. On the other hand $H_{\tau^{\prime}}$ acts differently. Indeed the occupancy of barrier is highly unfavoured in the ground state because of the large energy $W$. As a consequence the $W=0$ pair can jump from the 1 -st cluster to the $L-1$-th cluster an viceversa only at the fourth order in $H_{\tau^{\prime}}$. In this case the effective hopping through the barrier is $J e^{i \frac{4 \pi \phi}{\phi_{0}}}$ with

$$
J=-\frac{1}{2}\left(\tau^{\prime}\right)^{4} \frac{1}{|\Delta| \varepsilon^{2}} C
$$

where

$$
\varepsilon=E(3)+E(2)+E(1)+W
$$

(with $E(N)$ the ground state energy of the $\mathrm{CuO}_{4}$ with $N$ particles), and $\mathrm{C}$ is a order 1 costant given by

$$
\begin{gathered}
C=\left\langle\Psi_{L-1}(4)\left|\otimes\left\langle\Psi_{L}(0)\left|\otimes\left\langle\Psi_{1}(2)\left|H_{J}\right| \Psi_{1}(2)\right\rangle \otimes\right| \Psi_{L}(1)\right\rangle \otimes\right| \Psi_{L-1}(3)\right\rangle \times \\
\left\langle\Psi_{L-1}(3)\left|\otimes\left\langle\Psi_{L}(1)\left|\otimes\left\langle\Psi_{1}(2)\left|H_{J}\right| \Psi_{1}(3)\right\rangle \otimes\right| \Psi_{L}(0)\right\rangle \otimes\right| \Psi_{1}(3)\right\rangle \times \\
\left\langle\Psi_{L-1}(3)\left|\otimes\left\langle\Psi_{L}(0)\left|\otimes\left\langle\Psi_{1}(3)\left|H_{J}\right| \Psi_{1}(3)\right\rangle \otimes\right| \Psi_{L}(1)\right\rangle \otimes\right| \Psi_{L-1}(2)\right\rangle \times \\
\left\langle\Psi_{L-1}(2)\left|\otimes\left\langle\Psi_{L}(1)\left|\otimes\left\langle\Psi_{1}(3)\left|H_{J}\right| \Psi_{1}(2)\right\rangle \otimes\right| \Psi_{L}(0)\right\rangle \otimes\right| \Psi_{1}(4)\right\rangle,
\end{gathered}
$$

where $\left|\Psi_{\alpha}(N)\right\rangle$ is the ground state of the $\alpha$-th cluster with $N$ particles.

The resulting low-energy effective hamiltonian is then equivalent to a 1 -dimensional spin $1 / 2$ chain with $\mathcal{L}=L-1$ sites, given by (see Ref 16 for the case of $\tau^{\prime}=0$ ):

$$
H_{e f f}(\phi)=-2 \tau_{e f f} \sum_{\alpha=1}^{\mathcal{L}-1} S_{\alpha}^{z} S_{\alpha+1}^{z}+\tau_{e f f} \sum_{\alpha=1}^{\mathcal{L}-1}\left(S_{\alpha}^{+} S_{\alpha+1}^{-}+\text {h.c. }\right)-\tau_{e f f} S_{\mathcal{L}}^{z} S_{1}^{z}+\left(J e^{i \frac{4 \pi \phi}{\phi_{0}}} S_{\mathcal{L}}^{+} S_{1}^{-}+\text {h.c. }\right)
$$



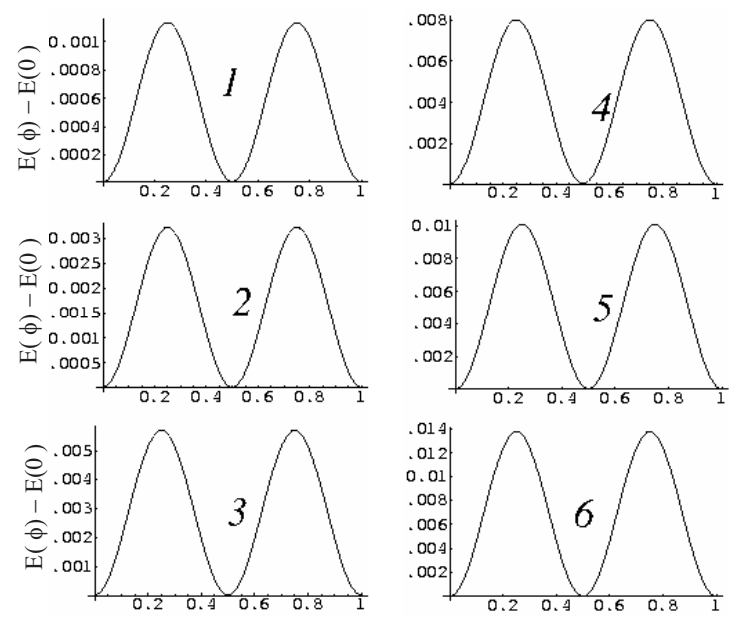

$\phi / \phi_{0}$

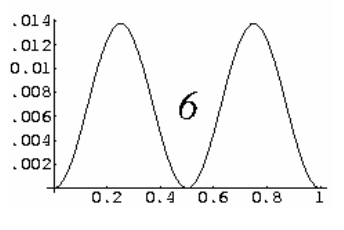

$\phi / \phi_{0}$

FIG. 7: $E(\phi)-E(0)$ (in units of $t_{p d}$ ) as a function of $\phi / \phi_{0}$ for $2 \times S_{t o t}^{z}=1,2,3,4,5,6$. Here we have taken and $J / \tau=0.1$.

We observe that the barrier has been "integrated out" and the resulting model has the 1 -st and the $\mathcal{L}$-th sites linked by $J$.

It is worth to note that this model, because of the the presence of two different parametres $\tau_{\text {eff }}$ and $J$, is not solvable by the Bethe ansatz, therefore we must resort numerical methods. We solved $H_{\text {eff }}(\phi)$ with $\mathcal{L}$ up to 12 and $S_{\text {tot }}^{z}$ up to 3. In this case, in the original fermionic model there were $L=13 \mathrm{CuO}_{4}$ clusters $(12+$ barrier $)$ and 6 $W=0$ pairs, that is $12 \times 2+6 \times 2=36$ fermionic particles. In other words the number of $W=0$ pairs is $2 \times S_{\text {tot }}^{z}$, which is a conserved quantity.

In figure we show the ground state energy $E(\phi)$ as a function of the magnetic flux for $\mathcal{L}=12$ and $2 \times S_{\text {tot }}^{z}=$ $1,2,3,4,5,6$. It is remarkable that whatever is the number of $W=0$ pairs in the original systems $(1,2,3,4,5,6)$, the peridicity of $E(\phi)$ does not change and shows the same superconducting flux quantization. This is a very interesting feature, suggesting that all the $W=0$ pairs may behave "coherently", providing the same macroscopic response, no matter of their number. It is also clear that the oscillations of $E(\phi)$ get more and more pronounced as the number of pairs in the ground state become of the same order of number of the lattice sites. This is an indication that such a behavior should survive in the thermodynamic limit.

Finally we observe that the trend of $E(\phi)$ by incresing the number of particles is deeply different with respect to the one observed in the repulsive Hubbard ring. In the latter case $\stackrel{910}{ }$, there are many minima between $\phi=0$ and $\phi=\phi_{0}$, depending on the ratio $N t / L U$, where $N$ is the number of particles and $L$ is the number of lattice sites. On the other hand, in the case under consideration it seems that the superconducting nature of the charge carriers "freezes" the period oscillations to be $\phi_{0} / 2$.

As a last remark, we notice that $E(\phi)$ is a smooth function of $\phi$. This means that no level crossing occurs between $\phi=0$ and $\phi=\phi_{0}$. This is a direct consequence of the breaking of the translational symmetry induced by the presence of the barrier. As discussed in the previous Section, this condition will be important in the time-dependent case, where, at least in the adiabatic approximation, the system at $t=0$ (supposed to be in the ground state at $\phi=0$ ) will be able to evolve continuosly to the static ground state at $\phi=\phi_{0} / 2$. This condition is necessary in order to have a time-dependent superconducting response.

\section{B. Time-Dependent Case}

Now we consider a time-dependent perturbation, with the effective hopping through the barrier given by $J e^{i 4 \pi V t}$. In this case we rely on the adiabatic approximation, such that, nevertheless of the time-dependence, the low energy Hamiltonian is the same as $H_{e f f}$, with $\phi / \phi_{0} \rightarrow V t$. The correctness of this approximation has been checked directly in the previous Section, in the time-dependent analysis of the single $\mathrm{CuO}_{4}$ cluster. There, the exact time-dependent solution of the whole original electronic problem was achieved and compared to the approximated one.

The exact time-dependent solution of the Schrödinger equation associated to $H_{\text {eff }}(t)$ is much harder than the static solution. Therefore we only consider the case $\mathcal{L}=4$ and $2 \times S_{\text {tot }}^{z}=2$. Anyway, in the original fermionic model, it means $L=5 \mathrm{CuO}_{4}$ clusters (4+barrier) and $2 W=0$ pairs, that is 12 particles. As initial condition at $t=0$ we take the gruond state of $H_{\text {eff }}(\phi)$ at $\phi=0$. 


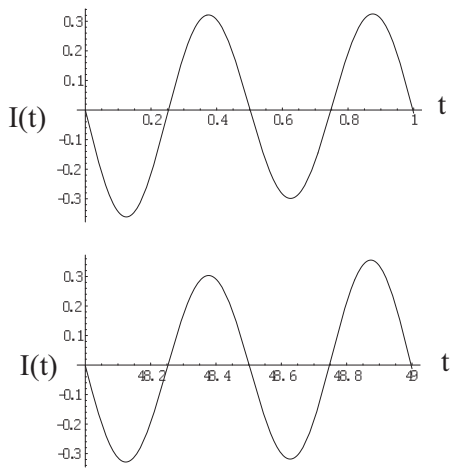

FIG. 8: $I(t)$ as a function of $V t$ for $2 \times S_{t o t}^{z}=2$ for two intervals $(0,1)$ and $(49,40)$ : Here we have taken and $J / \tau=0.1$.

Our aim is to evaluate the time-dependent current $I(t)$ through the barrier, i.e. through the bond governed by $J e^{i \frac{4 \pi V t}{\phi_{0}}}$, according to

$$
I(t)=J\left\langle\Psi(t)\left|e^{i 4 \pi V t} S_{\mathcal{L}}^{+} S_{1}^{-}-e^{-i 4 \pi V t} S_{\mathcal{L}}^{-} S_{1}^{+}\right| \Psi(t)\right\rangle .
$$

The exact numerical solution shows that $I(t)$ is a peridic function of $t$ with period $1 /(2 V)$. In figure we plot $I(t)$ as a function of $V t$ in two different unitary intervals: $(0,1)$ and $(48,49)$.

We stress that the time-dependent response of the many-units model have the same Josephson-like behaviour of the single-unit model.

\section{CONCLUSIONS}

We have shown how to model a Josephson-like junction using microscopic ingredients like electrons hopping in an atomic lattice, a tunneling barrier, and on-site correlation. The barrier consists of a bond characterized by a weak time-dependent hopping, where the emf is applied. The current response is easier to calculate and analyze when there is emf on a single bond. We stress that the Hubbard interaction $U$ leads to a current response in quantitative agreement with Eq. (1). This happens in circumstances that we understand, namely: i) the formation of $W=0$ pairs, ii) a well developed double minimum in the energy-flux plot, iii) a soft distortion of the symmetry opening a small gap (due to avoided crossing) in the many-body spectrum, iv) a small enough emf $V$, in order to allow for adiabatic response. When the last condition is realized, calculations based on the adiabatic approximation are in good agreement with the full solutions; this allows us to extend the analysis to fairly large systems. In modeling rings of Hubbard clusters, the barrier was formed by a $\mathrm{CuO}_{4}$ unit with high one-electron energy; in order to deal with the low-energy physics, we derived an effective Hamiltonian where the barrier is integrated out and represented by an effective, weakened, complex bond, similar to the barrier in the above distorted $\mathrm{CuO}_{4}$ case.

In the single-unit model, the superconducting AC current has the same frequency $\frac{2 \mathrm{eV}}{\hbar}$ as one would expect to observe in a macroscopic junction; in the latter case, however, the normal response is a DC current. In our cluster calculations, on the other hand, the current response of the normal systems is periodic with half the Josephson frequency; this happens with one-particle systems and with 4 -body ones with $U=0$, when no pairing occurs. This is due to a size effect that we wish to comment upon here.

As shown in Ref. (11), in the non-interacting case (which is clearly normal) the transient current across a tunnel barrier between infinite leads tends to a constant for long times; the asymptotic $t \rightarrow \infty$ behavior yields the currentvoltage characteristics calculated by several people by time-independent approaches 12131415 . This has recently been proven as a theorem by Stefanucci and Almbladh 7 within the Time-Dependent Density Functional Theory in the Local Density Approximation. In order to understand the difference, we recall that the infinite junctions considered in $(11)$ never reach equilibrium, and the steady current that holds in the $t \rightarrow \infty$ limit is just a never-ending transient. On the contrary, the oscillating behavior of the small cluster follows almost adiabatically the instantaneous ground state. Another difference is that the normal system is unable to trap the magnetic flux: when the field penetrates the sample our modeling should be less appropriate than in superconductors. So we can expect that the normal current oscillation at half frequency, that we observe in the small cluster, should acquire a longer and longer transient behaviour with increasing sample size, while the field penetration should superimpose different oscillation frequencies. 
Instead, the superconducting pairs should keep their frequency in line with equation (11) right to the thermodynamic limit.

According to our interpretation, the Josephson effect is essentially another facet of the superconducting flux quantization. The necessary distortion is always present in macroscopic systems, where in addition a large energy separates the states of different pair symmetry. Unbiased supercurrents and Shapiro spikes are also obtained by the same models that mimic the AC Josephson behaviour.

In the many-units model, the $\mathrm{CuO}_{4}$ clusters are combined in large systems linked by weak hopping integrals; the solution is found by mapping the problem into a spin $1 / 2$ chain. In the time-independent case, we presented evidence that the flux quantization pattern is unmodified when several bound pairs propagate together, except that the energy barriers separating the minima grow large. In the time-dependent case we solved Schrödinger equation numerically, and again we got a Josephson-junction-like behavior.

Finally, it is remarkable that even with one pair, that can be on the left or on the right, there is enough uncertainty on the pair number to create some ghost order parameter.

\section{Acknowledgements}

E. P. was supported by INFN grant 10068 .

1 B. D. Josephson, Phys. Letters 1, 251 (1962)

2 G. Grosso and G. Pastori Parravicini, Solid State Physics, Academic Press, San Diego (2000).

3 J. G. Bednorz and K. A. Müller, Z. Phys. B 64, 189 (1986).

4 R. A. Ferrel and R. E. Prange, Phys. Rev. Letters 10, 479 (1963)

5 A. Balzarotti, M. Cini, E.Perfetto and G.Stefanucci, J. Phys.: Condens. Matter 16 (2004) R1387-R1422.

6 W. Kohn, Phys. Rev. 133 A171 (1964).

7 G. Stefanucci and C.-O. Almbladh, Phys. Rev. B69 195318 (2004)

8 A. Callegari, M. Cini, E. Perfetto and G. Stefanucci, Eur. Phys. J.B 34, 455 (2003).

${ }^{9}$ N. Yu and M. Fowler, Phys. Rev.B45 11795 (1992).

10 F. V. Kusmartsev, Phys. Rev.B52 14445 (1995).

11 M. Cini, Phys. Rev.B22 5587 (1980).

12 R. Landauer, IBM J. Res. Dev. 1, 233 (1957)

13 C. Caroli, R. Combescot, P. Noziéres and D. Saint-James, J. Phys. C 4, 916 (1971)

14 T. E. Feuchtwang, Phys. Rev. B12, 3979 (1975)

15 Y. Meir and N. S. Windgreen, Phys. Rev. Lett. 68, 2512 (1992)

16 A. Callegari, M. Cini, E. Perfetto and G. Stefanucci, Eur. Phys. J. B 34, 455 (2003). 